\title{
Assessing Dysplasia of a Bronchial Biopsy with FTIR Spectroscopic Imaging
}

\author{
Liberty Foreman ${ }^{\mathrm{a}}$, James A. Kimber ${ }^{\mathrm{b}}$, Katherine V. Oliver ${ }^{\mathrm{a}}$, James M. Brown ${ }^{\mathrm{c}}$, Samuel M. Janes ${ }^{\mathrm{c}}$, \\ Tom Fearn ${ }^{\text {d, Sergei G. Kazarian**b }}{ }^{*}$ Peter Rich*a \\ ${ }^{a}$ Glynn Laboratory of Bioenergetics, Institute of Structural and Molecular Biology, University \\ College London, Gower Street, London, WC1E 6BT, U.K. ${ }^{b}$ Department of Chemical Engineering, \\ Imperial College London, South Kensington Campus, London, SW7 2AZ, U.K. ${ }^{c}$ Lungs for Living \\ Research Centre, UCL Respiratory, University College London, London, U.K. ${ }^{\mathrm{d} D e p a r t m e n t ~ o f ~}$ \\ Statistical Science, University College London, 1-19 Torrington Place, WC1E 7HB, U.K. \\ *prr@ucl.ac.uk,**s.kazarian@imperial.ac.uk
}

\begin{abstract}
An FTIR image of an $8 \mu \mathrm{m}$ section of de-paraffinised bronchial biopsy that shows a histological transition from normal to severe dysplasia/squamous cell carcinoma (SCC) in situ was obtained in transmission by stitching together images of 256 x $256 \mu \mathrm{m}$ recorded using a 96 x 96 element FPA detector. Each pixel spectrum was calculated from 128 co-added interferograms at $4 \mathrm{~cm}^{-1}$ resolution. In order to improve the signal to noise ratio, blocks of $4 \times 4$ adjacent pixels were subsequently averaged. Analyses of this spectral image, after conversion of the spectra to their second derivatives, show that the epithelium and the lamina propria tissue types can be distinguished using the area of troughs at either 1591, 1334, 1275 or $1215 \mathrm{~cm}^{-1}$ or, more effectively, by separation into two groups by hierarchical clustering (HCA) of the 1614-1465 region. Due to an insufficient signal to noise ratio, disease stages within the image could not be distinguished with this extent of pixel averaging. However, after separation of the cell types, disease stages within either the epithelium or the lamina propria could be distinguished if spectra were averaged from larger, manually selected areas of the tissue. Both cell types reveal spectral differences that follow a transition from normal to cancerous histology. For example, spectral changes that occurred in the epithelium over the transition from normal to carcinoma in situ could be seen in the $1200-1000 \mathrm{~cm}^{-1}$ region, particularly as a decrease in the second derivative troughs at 1074 and $1036 \mathrm{~cm}^{-1}$, consistent with changes in some form of carbohydrate. Spectral differences that indicate a disease transition from normal to carcinoma in the lamina propria could be seen in the $1350-1175 \mathrm{~cm}^{-1}$ and $1125-1030 \mathrm{~cm}^{-1}$ regions. Thus demonstrating that a progression from healthy to severe dysplasia/squamous cell carcinoma (SCC) in situ can be seen using FTIR spectroscopic imaging and multivariate analysis.
\end{abstract}

Keywords: FTIR spectroscopic imaging, FTIR, lung cancer, bronchial tissue, multivariate analysis.

\section{INTRODUCTION}

Lung cancer is the most common cancer worldwide with over 1.8 million cases diagnosed in 2012 alone. ${ }^{1}$ With an estimated 4300 deaths per day, only one third of all diagnosed lung cancer cases survive one year, dropping to less than $15 \%$ for a five year survival. ${ }^{1}$ However, if the disease is identified at the early stage the one year survival rate increases to $56-73 \% .^{2}$

The gold standard for diagnosing lung cancer is by histological analyses of biopsies, which is more accurate than other tests, for example cytology or analysis of sputum. ${ }^{3}$ There are several different techniques that can be used to biopsy the lung but obtaining a biopsy adequate for histological diagnoses can be difficult as the biopsy must be physiologically intact. Some techniques can cause damage to the biopsies making them unsuitable for histological analyses. However, this is not identified until the biopsy has been processed, which typically takes a couple of weeks.

Fourier transform infrared (FTIR) and Raman vibrational spectroscopies are being increasingly investigated as possible diagnostic tools for a variety of diseases. ${ }^{4-6}$ They can provide quantitative information on cellular changes in DNA, protein, carbohydrates and other metabolites. ${ }^{4,5}$ Both FTIR, ${ }^{7-12}$ and Raman ${ }^{13-15}$ spectroscopic methods have been applied to lung cancer diagnoses. FTIR studies have included, single element attenuated total reflection, ATR-FTIR, ${ }^{8,9,12}$ transmission

Optical Diagnostics and Sensing XV: Toward Point-of-Care Diagnostics, edited by Gerard L. Coté, Proc. of SPIE Vol. 9332, 93320S · C 2015 SPIE · CCC code: 1605-7422/15/\$18 · doi: 10.1117/12.2078966 
micro-FTIR spectroscopic imaging ${ }^{7,10}$ and synchrotron FTIR spectroscopy. ${ }^{11}$ Single element ATR-FTIR studies have included distinguishing healthy from cancerous lung samples with fresh tissue, ${ }^{8}$ pulverised tissue ${ }^{12}$ and serum. ${ }^{9}$ Raman probes have been used to investigate in vivo diagnosis in the lung in a small number of patients, ${ }^{13,15}$ with differences found between heathy and cancerous tissue. Raman spectroscopy has also been used to identify changes between healthy and cancerous lung epithelial cell lines. ${ }^{14}$ However, high equipment/operational costs, to date, precluded development of these methods into a routine clinical diagnostic tool. The use of spectroscopy in medical diagnostics could be advantageous as it can produce, rapid quantitative results that do not rely on the biopsy being physiologically intact.

In this study FTIR spectroscopic imaging in transmission was used to characterise cell and disease progression of lung squamous cell carcinoma (SCC) in a single deparaffinised $8 \mu \mathrm{m}$ biopsy section that contains histologically-defined areas of disease progression. Disease stages present include healthy, mild/moderate/severe dysplasia and squamous cell carcinoma (SCC) in situ. The use of such a sample eliminates inter-sample and inter-patient differences that might be unrelated to carcinogenesis. It is rare for a single sample to display a complete disease transition from healthy to carcinoma in situ, and to the best of our knowledge, FTIR spectroscopic imaging of such a sample has not been described in the literature.

\section{MATERIALS AND METHODS}

\subsection{Sample preparation}

A bronchial biopsy sample was excised from a patient with pre-invasive SCC. The patient was participating in a clinical research study approved by the local ethics committee (06/Q0505/12) and routine informed consent was taken prior to the procedure. A biopsy was taken from the right upper lobe as part of the undergoing surveillance of the patients known bronchial pre-invasive lung cancer. It was fixed in $4 \%$ formaldehyde solution for 12 hours, and embedded in paraffin wax using a standard protocol. ${ }^{16}$ An approximately $4 \mathrm{~mm} \times 1 \mathrm{~mm}, 4 \mu \mathrm{m}$ thick section was cut for H\&E staining and analysis by an expert histopathologist at University College London Hospital (UCLH), UK. Figure 1A shows the tissue annotated by the histopathologist. It contains healthy epithelia, mild dysplasia, moderate dysplasia and severe dysplasia/carcinoma in situ as well as the underlying lamina propria. For the FTIR spectroscopic image, an additional $8 \mu \mathrm{m}$ section was cut and mounted on a $2.5 \mathrm{~mm}$ thick $\mathrm{CaF}_{2}$ window. The sample was deparaffinised with hexane and washed with ethanol/water mixtures of; $100 \%, 95 \%, 70 \%$ and $50 \%$ for 2 minutes per solution. It was stored in a container with desiccant before the FTIR image was recorded.

\subsection{FTIR spectroscopic measurement parameters}

An IFS 66/s spectrometer (Bruker) coupled to a Hyperion 96 x 96 FPA detector was used to record the spectra in transmission mode at Imperial College London (ICL), UK. Data in the 4000-900 $\mathrm{cm}^{-1}$ region were collected, but only the $1800-900$ $\mathrm{cm}^{-1}$ region was analysed in detail. Spectra from each FPA element were averaged from 128 co-added interferograms at $4 \mathrm{~cm}^{-1}$ resolution. Where possible, a white light image of the same sample area was also recorded. In order to map the whole $4 \mathrm{~mm}$ x $1 \mathrm{~mm}$ sample, a succession of 96 x 96 pixel arrays measuring $256 \mu \mathrm{m}$ x $256 \mu \mathrm{m}$, were automatically recorded and subsequently stitched together to produce one image of the whole sample with a 16 × 3 tile array (i.e. a 1536 x 288 pixel image). After acquiring the image, the spatial resolution was decreased by binning blocks of $4 \times 4$ pixels in order to decrease computational expenditure and to improve the signal to noise ratio. The projected pixel size at the original resolution was $2.7 \mu \mathrm{m} \times 2.7 \mu \mathrm{m}$, which was reduced to $10.8 \mu \mathrm{m} \times 10.8 \mu \mathrm{m}$ when binned.

\subsection{Spectral pre-processing and analysis parameters}

All spectral processing was performed in MATLAB R2013b. Spectral contributions from water and water vapour were removed by fractional subtraction of pre-recorded reference model spectra. Spectra that had an amide II protein contribution (measured as the maximum difference between peak and trough in the $1562-1468 \mathrm{~cm}^{-1}$ range) above $0.05 \Delta \mathrm{A}$ were selected for further analyses. They were first normalised to the mean amide II absorbance of 0.15 before conversion to second derivative with 17 point Savitzky-Golay smoothing. MATLAB PLS toolbox R7.9.3 was used for hierarchical clustering (HCA) and principal component (PCA) analyses. HCA was performed with Ward's algorithm on the 1614-1465 $\mathrm{cm}^{-1}$ spectral region after mean centring. PCA was performed after mean centring and with no threshold set. Models built with 1-10 principal components were tested. Three components were chosen for all PCA models, this was based on selecting the number of components where diminishing returns were reached in a cumulative variance plot. Both PCA 
models captured $99 \%$ of variation with three components. The resulting PC loadings were analysed to ensure that the they were consistent with IR signatures expected to arise from biochemical changes associated with disease progression.

\section{RESULTS}

\subsection{Cell type differentiation}

Figure 1A shows the H\&E stained section, together with the histologically defined regions of epithelium and lamina propria, and the areas of epithelial disease stage progression.

It is well documented that different cell types can have different FTIR characteristics. ${ }^{17,18}$ The spectral differences between surface epithelial cells and the underlying lamina propria of a bronchial biopsy are shown in Figure 1B. These two cell types were easily separated by integration of the 1591 (Figure 1C), 1334 (Figure 1D), 1215 and $1275 \mathrm{~cm}^{-1}$ bands. HCA of the 1614-1465 $\mathrm{cm}^{-1}$ region could also be used to separate them (Figure 1E). This same region has been used to successfully separate oesophageal epithelial and lamina propria cell types by HCA, and with similar spectral features (Foreman et. al unpublished work). Differentiation of the two bronchial cell types could be achieved by HCA with other spectral regions.

\subsection{Disease stage differentiation}

\subsubsection{Analysis of the epithelium}

The effects of disease progression on cellular IR characteristics were investigated. The epithelium and the lamina propria regions were analysed separately in order to eliminate spectral differences arising from cell type (above). However, the signal:noise ratio of a $4 \times 4$ binned image, where each pixel would have an approximate size of $10.8 \mu \mathrm{m}^{2}$, was insufficient to accurately de-convolute the very small signal differences that distinguish different disease stages. Therefore, fifteen regions were manually selected along the epithelium (Figure 2A and 2B). Regions 1-15 contain the following number of pixels at the original resolution with a projected pixel size of $2.7 \mu^{2}$ : region 1, 2224; region 2, 992; region 3, 1392; region 4, 1344; region 5, 880; region 6, 2144; region 7, 3744; region 8, 3376; region 9, 2992; region 10, 4720; region 11, 5056; region 12, 2416; region 13, 2720; region 14, 2352 and region 15, 3280. According to the histopathology, regions 1-3 were healthy, regions 4-6 exhibited mild dysplasia, regions 7-9 showed moderate dysplasia and regions 10-15 showed severe dysplasia/carcinoma in situ. The spectra within each of these regions were averaged and the averaged spectra were compared after normalisation of their amide II intensities (Figure 2C and 2D). Spectral differences were evident, most clearly seen in the second derivative troughs at 1163,1074 and $1036 \mathrm{~cm}^{-1}$ decreased as dysplasia progressed, and where the peak at $1095 \mathrm{~cm}^{-1}$ also decreased as well as downshifting to $1093 \mathrm{~cm}^{-1}$.

Figure 2A and 2B show the integrals of second derivative troughs 1163 and $1036 \mathrm{~cm}^{-1}$, respectively. The difference between healthy (regions 1-3) and the diseased (regions 4-15) epithelial regions can be seen clearly in the colour change from red/yellow to blue. The transition from healthy to mild dysplasia (region 4) is reflected well in the $1036 \mathrm{~cm}^{-1}$ trough (Figure 2A) where the integral 0.009 was in-between the average healthy integral 0.0014 and the dysplastic/ carcinoma in situ integral of 0.007 . The $p$-value from a Man-Whitney $\mathrm{U}$ test between healthy and dysplastic/carcinoma in situ was 0.0044 for both the 1163 and $1036 \mathrm{~cm}^{-1}$ integrals, making the difference between healthy and dysplastic significant. However, no significant difference was found when comparing the integrals of $1163,1095,1074$ or $1036 \mathrm{~cm}^{-1}$ between mild, moderate and severe dysplasia/carcinoma in situ.

A PCA with three components was performed on the 1100-1030 $\mathrm{cm}^{-1}$ spectral region (Figure 3). PC 1 separates healthy from the other dysplastic regions, and PC 3 separates moderate dysplastic regions from the severe dysplastic regions. However, there is no clear resolution of the mild dysplastic regions (Figure 3A). It was still possible to retain good separation of disease progression by averaging spectra after subdividing each region into two, but clear separation was lost with smaller subdivision due to degradation of signal to noise. 

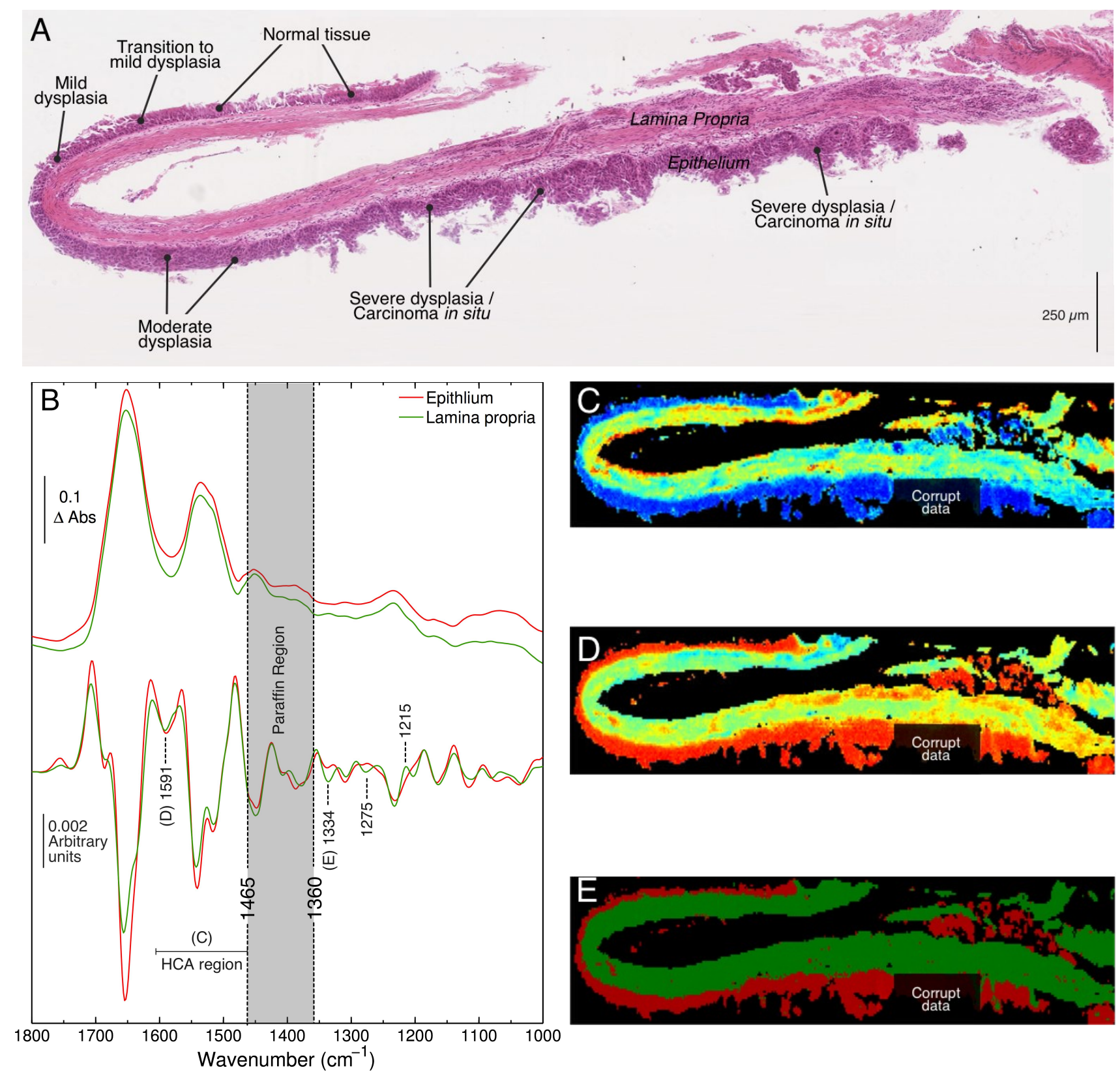

Figure 1: Identification of cell types and disease stages of a bronchial biopsy section. A) Histopathological analysis of the H\&E stained section of the bronchial sample. B) Extracted spectra showing the absorbance (top) and second derivative (bottom) spectra of the epithelium (red) and lamina propria (green), derived by averaging the HCA-defined regions of Figure 1E. The grey area indicates the region in which paraffin absorbs. C) Heat map that shows epithelium (blue) and lamina propria (yellow/red) separated on the size of the integral of the $1591 \mathrm{~cm}^{-1}$ trough. D) Heat map that shows epithelium (red) and lamina propria (yellow/green) separated on the size of the integral of the $1334 \mathrm{~cm}^{-1}$ trough. E) Diagrammatical representation of the two major groups identified in the HCA of the 1614-1465 $\mathrm{cm}^{-1}$ region. Epithelium is shown in red and lamina propria in green. The Corrupt data box present in C), D) and E) indicate two adjacent tiles of the FTIR image where the data recorded were corrupt and unusable. 

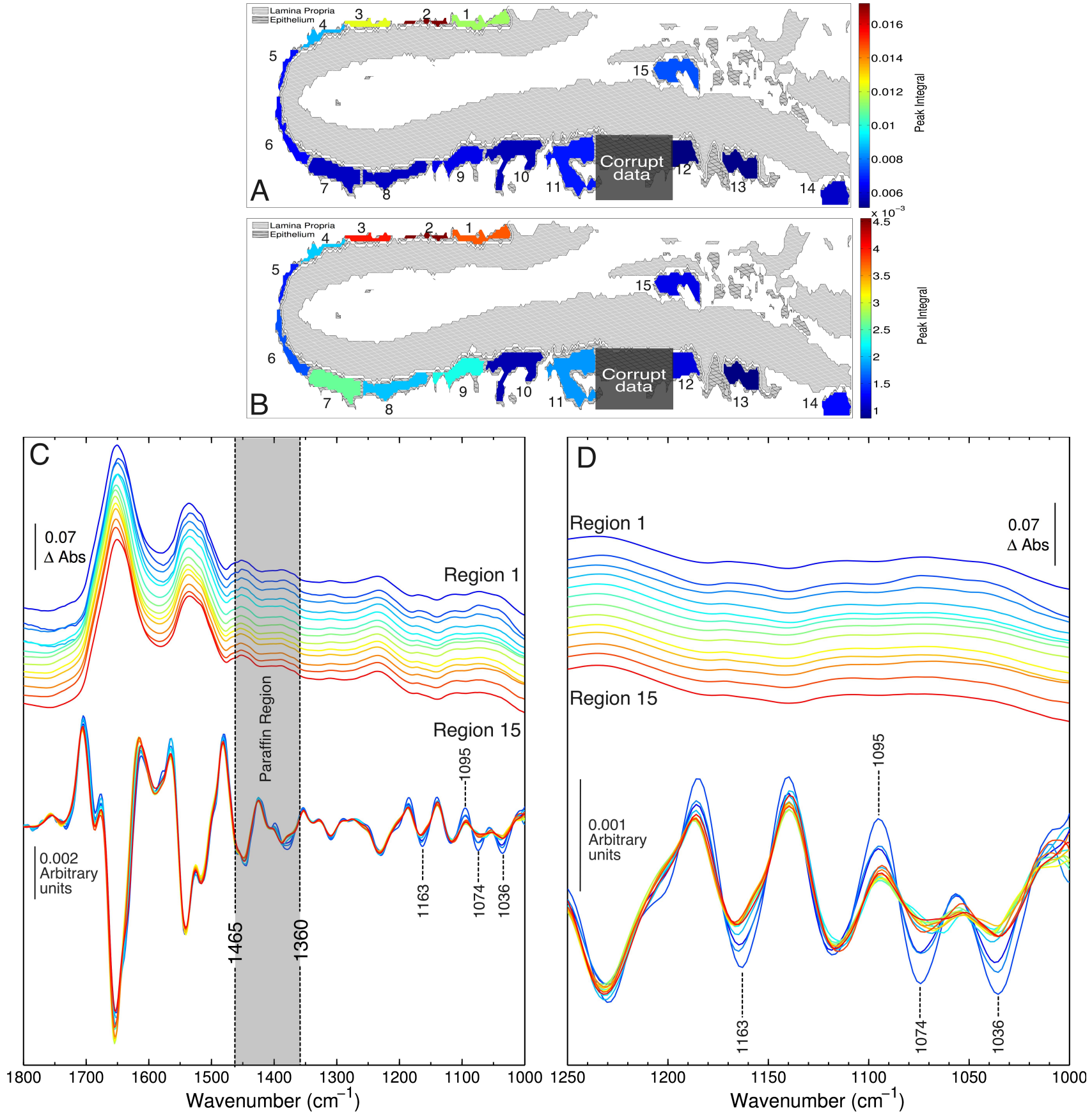

Figure 2: FTIR Spectra from bronchial epithelium at different disease stages. Fifteen regions were selected with the following disease stages: regions 1-3, healthy; 4-6, mild dysplasia, regions 7-9, moderate dysplasia and regions 10-15, severe dysplasia/carcinoma in situ. A) The regions were colour coded according to the size of the $1036 \mathrm{~cm}^{-1}$ second derivative trough integral. B) The regions were colour coded according to the size of the $1163 \mathrm{~cm}^{-1}$ second derivative trough integral. The Corrupt Data box present in A) and B) indicate two adjacent tiles of the FTIR image where the data recorded were corrupt and unusable. C) Shows the absorbance (top) and second derivative (bottom) spectra in the $1800-1000 \mathrm{~cm}^{-1}$ region, averaged from the 15 selected epithelial regions ranging from healthy (blue) to severe dysplasia/carcinoma in situ (red). D) The $1250-1000 \mathrm{~cm}^{-1}$ region of the same spectra 


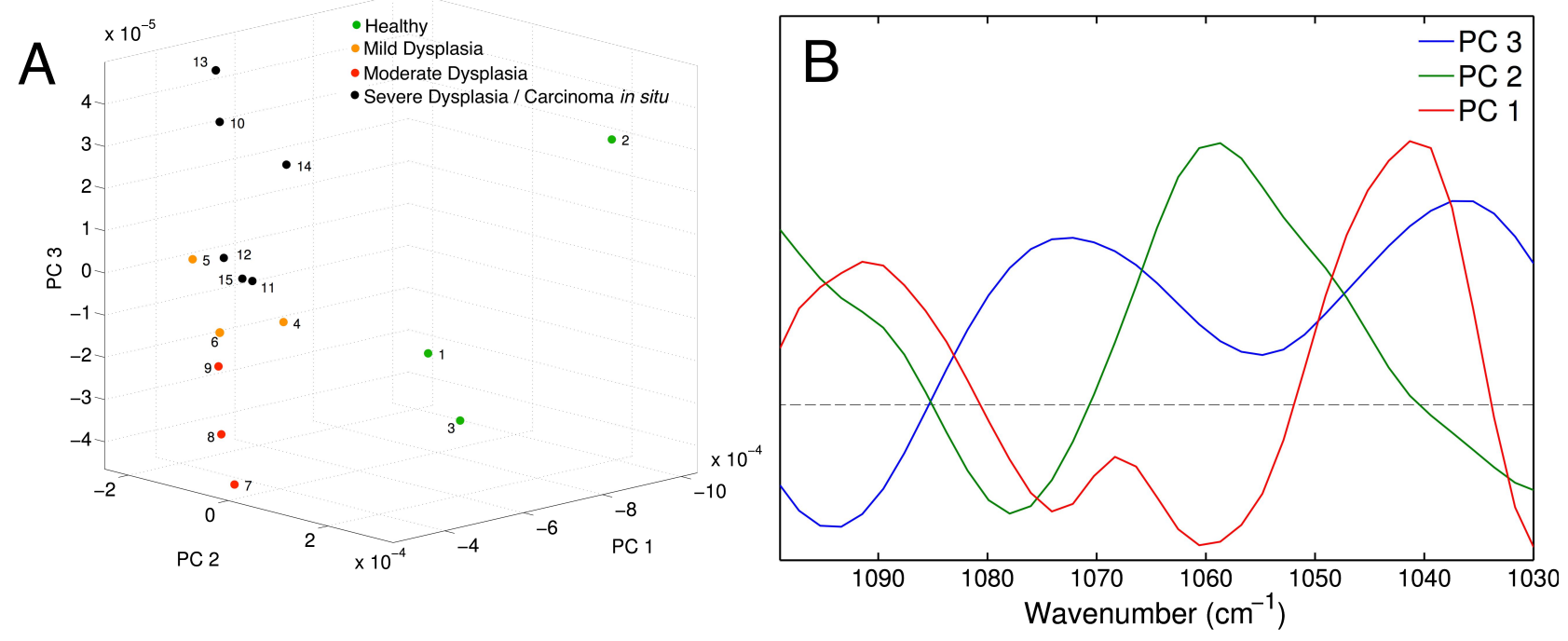

Figure 3: PCA of the spectra from the manually selected bronchial epithelium regions. A) Scatter plot showing the PCA scores from the first three components of a PCA performed using the $1100-1030 \mathrm{~cm}^{-1}$ region. Each data point is labelled with its corresponding region number: green, healthy; orange, mild dysplasia; red, moderate dysplasia; black, severe dysplasia/carcinoma in situ. B) The corresponding PCA loadings. 


\subsubsection{Analysis of the lamina propria}

Since SCC originates in the surface epithelial cells, the major spectral differences are expected to arise in this region. In order to investigate whether the progression of dysplasia also affected spectral properties of the underlying tissue, fifteen lamina propria regions were manually selected from the mapped FTIR image (Figure 4). Figure 5 shows the averaged absolute (top) and second derivative (bottom) spectra from these 15 regions. Regions 1-15 contain the following number of pixels at the original resolution with a projected pixel size of $2.7 \mu \mathrm{m}^{2}$ : region 1,9360 ; region 2, 5232; region 3, 5264; region 4, 5008; region 5, 3376; region 6, 4896; region 7, 5536; region 8, 6544; region 9, 6288; region 10, 7872; region 11, 6576; region 12, 9072; region 13, 9296; region 14, 10704 and region 15, 4288. According to the histopathology, the regions were adjacent to the following epithelium: regions 1-3, healthy; regions 4-6, mild dysplasia; regions 7-9, moderate dysplasia and regions 10-15, severe dysplasia/carcinoma in situ. Spectral differences were evident in these averaged spectra, seen clearly in the second derivative troughs at $1334 \mathrm{~cm}^{-1}$ and $1279 \mathrm{~cm}^{-1}$ and peaks at $1215 \mathrm{~cm}^{-1}$ and $1066 \mathrm{~cm}^{-1}$, which decreased in intensity as disease progressed. The $1279 \mathrm{~cm}^{-1}$ band and the $1080-1050 \mathrm{~cm}^{-1}$ spectral region also exhibited some shifts as the disease stage progresses (Figure 5A and 5B). Figure 4A-D shows the second derivative integrals of the 1334, 1279 1066 and $1215 \mathrm{~cm}^{-1}$ band respectively. The integrals of 1334,1279 and $1066 \mathrm{~cm}^{-1}$ show a gradual transition from healthy through to severe dysplasia/carcinoma in situ. The $1215 \mathrm{~cm}^{-1}$ (Figure 4D) integral shows a significant difference between healthy and dysplastic states, although there is no significant difference between the stages of dysplasia.

The regions of the spectra that contain the most significant differences between disease stages in the lamina propria are those from 1350-1196 $\mathrm{cm}^{-1}$ and 1097-1041 $\mathrm{cm}^{-1}$. Figure 6A shows a scatter plot of the first 3 principal component scores of the 15 selected regions. The same resolution of differences could be seen when the areas selected for averaging spectra were subdivided by a factor of four but was lost with further subdivision.

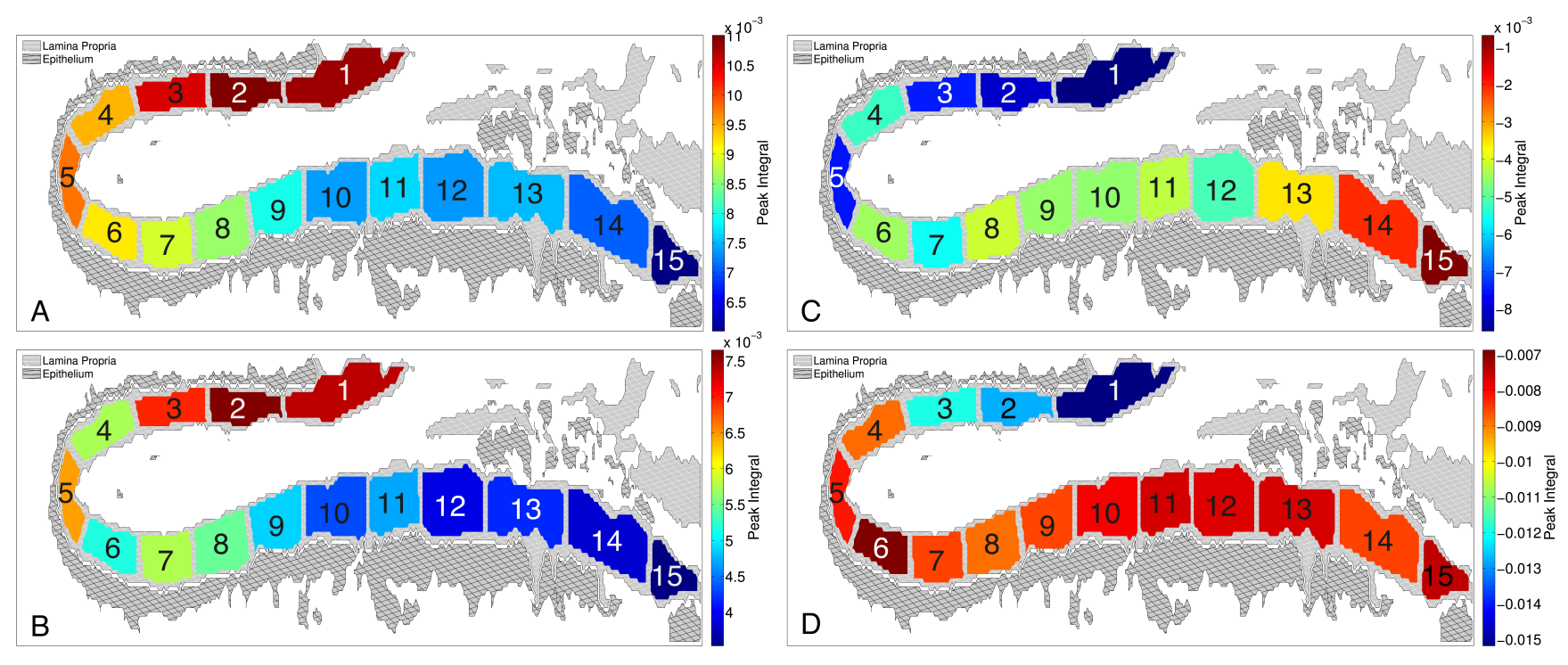

Figure 4: Peak integrals of spectral features from the manually selected regions of the bronchial lamina propria. Regions of the lamina propria colour were coded to the integrals of features at: A) $1334 \mathrm{~cm}^{-1}$;B) $1279 \mathrm{~cm}^{-1}$; C) $1066 \mathrm{~cm}^{-1}$; D) 1215 $\mathrm{cm}^{-1}$. The regions were adjacent to the following epithelium: regions 1-3, healthy; regions 4-6, mild dysplasia; regions 7-9, moderate dysplasia and regions 10-15, severe dysplasia/carcinoma in situ. 

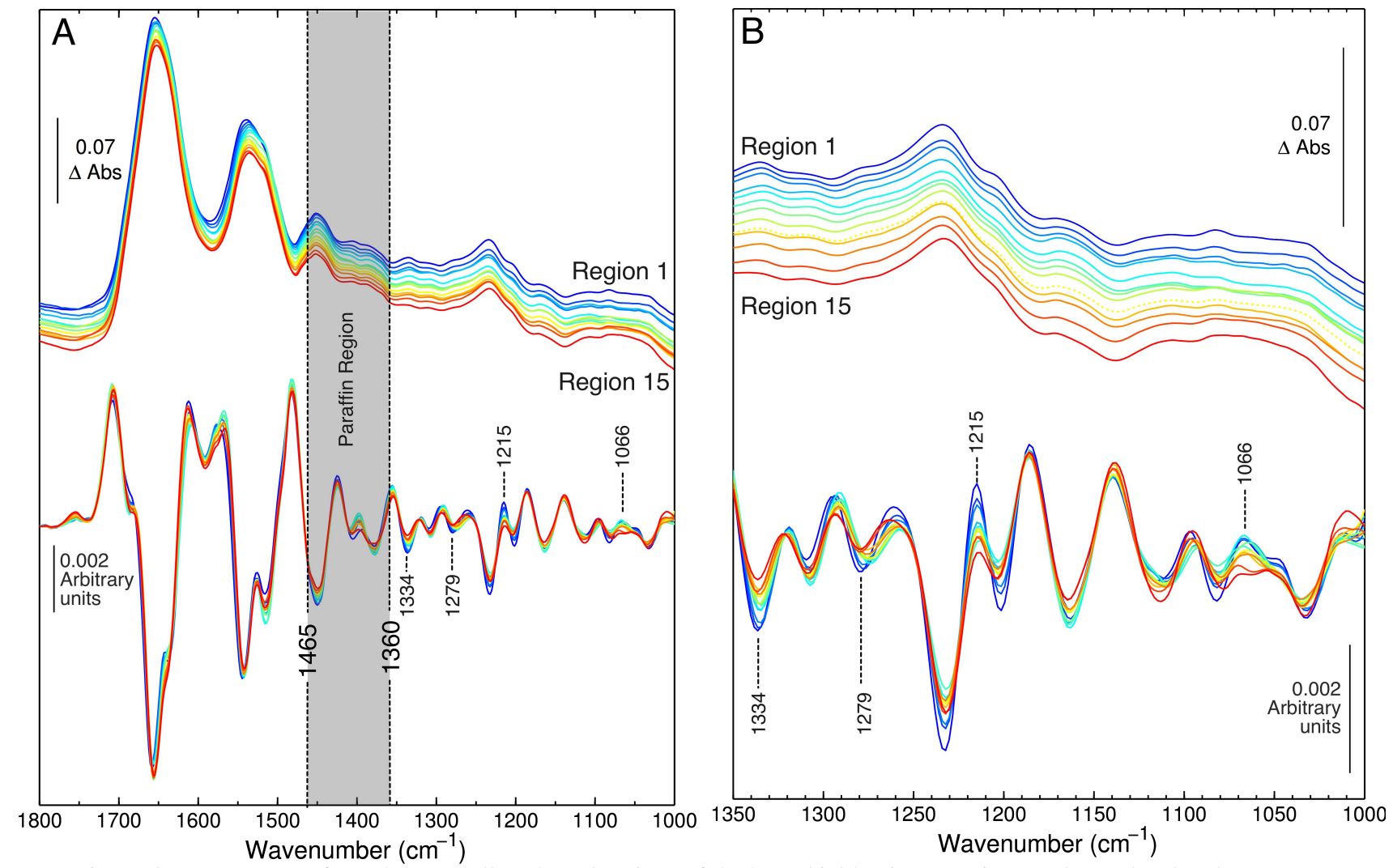

Figure 5: FTIR Spectra from the manually selected regions of the bronchial lamina propria. A) Shows the absorbance (top) and second derivative (bottom) spectra in the $1800-1000 \mathrm{~cm}^{-1}$ region, averaged from 15 regions ranging from healthy (blue) to severe dysplasia/carcinoma in situ (red). B) The $1250-1000 \mathrm{~cm}^{-1}$ region of the same spectra. The position of the manually selected regions from lamina propria can be seen in Figure 4.
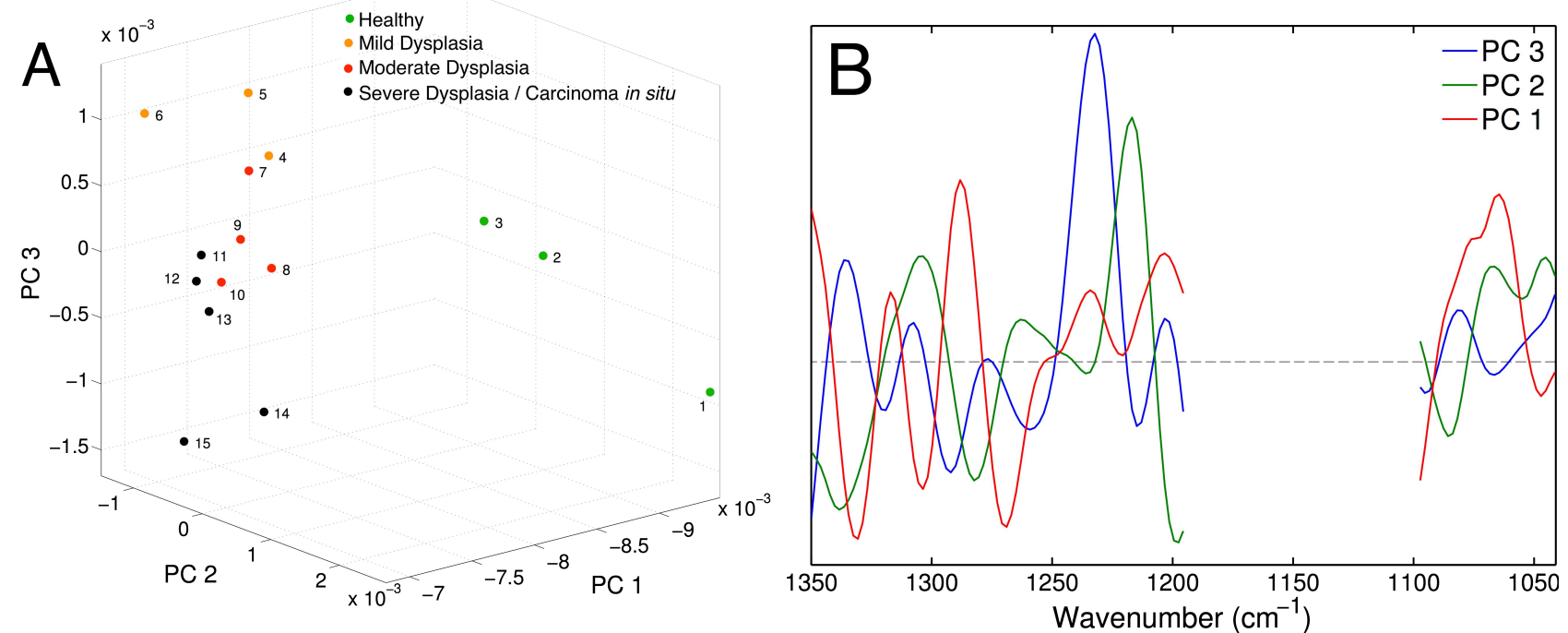

Figure 6: PCA of the spectra from the bronchial lamina propria regions adjacent to the diseased epithelium. A) Scatter plot of the PCA scores from the first three components of a PCA of the 1350-1196 and 1097-1041 $\mathrm{cm}^{-1}$ regions. Each data point is labelled with its corresponding region number: green, healthy; orange, mild dysplasia; red, moderate dysplasia; black,severe dysplasia/ carcinoma in situ. B) The corresponding PCA loadings. 


\section{DISCUSSION}

As the spectral differences between the epithelium and lamina propria of the bronchial sample are greater that the differences between disease stages, these two cell types were first separated and then analysed separately for changes associated with disease stage. This could be achieved easily by integration of bands at $1591,1334,1215$ and $1275 \mathrm{~cm}^{-1}$, or by HCA of the 1614-1465 $\mathrm{cm}^{-1}$ region. Differences in this latter region are similar to those found between the epithelium and lamina propria regions of oesophagus (Foreman et al. unpublished work). Lamina propria is a structural tissue comprised of a network of fibrous tissue that contains significantly more collagen and blood vessels than the epithelium, ${ }^{19}$ which when healthy is a thin layer of cells. There will, therefore, be more nuclei present in the epithelium and this might be one factor that accounts for its higher absorbance in the $1150-1000 \mathrm{~cm}^{-1}$ region since DNA is well known to contribute in this range. ${ }^{20}$

Analysis of second derivative spectra from the selected regions of the epithelium at different disease stages has revealed troughs at 1163,1074, 1036 and a peak $1095 \mathrm{~cm}^{-1}$ that decrease in intensity with each disease stage. The $1095 \mathrm{~cm}^{-1}$ band also exhibits a $2 \mathrm{~cm}^{-1}$ decrease in the peak position. This region is commonly attributed to changes in DNA and metabolites. Glucose and/or related carbohydrate compounds are likely to be the main contributors to the 1074 and 1036 $\mathrm{cm}^{-1}$ bands. A recent biochemical study on a lung cancerous cell line versus a healthy cell line by Chaudhri et al. ${ }^{21}$ suggests that there is a decrease in metabolites including glucose, supporting our finding. In the epithelium the biggest spectral difference was between the normal cells and the diseased cells. However, a PCA showed that a principal component that resembles glucose changes might be used to distinguish mild/moderate dysplasia and severe dysplasia/carcinoma in $s i t u$. This difference remains resolved even when the size of the manually selected regions are reduced.

It might be expected that the changes in the epithelium would be more significant those in the lamina propria as dysplasia arises in the epithelium. However, analysis of manually selected regions in the lamina propria also showed spectral changes associated with different disease stages. The second derivative peaks 1215 and $1066 \mathrm{~cm}^{-1}$ and troughs 1334 and 1279 $\mathrm{cm}^{-1}$, all decrease as the severity of dysplasia increases. The $1066 \mathrm{~cm}^{-1}$ region is of particular interest, as there appears to be an introduction of one or more components. It is known that cancer development triggers the inflammatory response, ${ }^{22,23}$ and so it is possible that these additional components and the changes in the position of the bands (relative to protein) are attributed to the introduction of leukocytes and other cells/proteins recruited to the area in response to the inflammatory process.

In conclusion, we have analysed a SCC bronchial biopsy section by micro-FTIR spectroscopic imaging where cell and disease type were identified using an adjacent H\&E section analysed by an expert histopathologist at University College London Hospital (UCLH), UK. The tissue displays a transition from healthy to cancerous, which includes mild, moderate and severe dysplasia as well as carcinoma in situ. The sample also includes both the surface epithelium and the underlying lamina propria. We have identified spectral features that can be used to differentiate the epithelium and lamina propria $\left(1614-1465,1334,1215\right.$ and $\left.1275 \mathrm{~cm}^{-1}\right)$ and further differences that can be used to partially identify all disease stages within both of the two tissue types.

\section{ACKNOWLEDGMENTS}

We are grateful to the Medical Research Council and Roy Castle Lung Cancer Foundation (JB and SMJ) for funding this research. SMJ is a Wellcome Senior Fellow in Clinical Science

\section{REFERENCES}

[1] Ferlay, J., Soerjomataram, I., Ervik, M., Dikshit, R., Eser, S., Mathers, C., Rebelo, M., Parkin, D. M., Forman, D. and Bray, F., "GLOBOCAN 2012 v1.0, Cancer Incidence and Mortality Worldwide: IARC CancerBase No. 11 [Internet]," Lyon, France: International Agency for Research on Cancer (2012).

[2] Peake, M. D., Chanarin, N. and Stanley, R. A., "The National Lung Cancer Audit (LUCADA) programme in England: C8-07," Journal of Thoracic ... (2007).

[3] Ofiara, L. M., Navasakulpong, A., Beaudoin, S. and Gonzalez, A. V., "Optimizing tissue sampling for the diagnosis, subtyping, and molecular analysis of lung cancer," Frontiers in oncology 4, 253-253 (2014).

[4] Kendall, C., Isabelle, M., Bazant-Hegemark, F., Hutchings, J., Orr, L., Babrah, J., Baker, R. and Stone, N., "Vibrational spectroscopy: a clinical tool for cancer diagnostics," The Analyst 134(6), 1029-1045 (2009). 
[5] Diem, M., Miljković, M., Bird, B., Chernenko, T., Schubert, J., Marcsisin, E., Mazur, A., Kingston, E., Zuser, E., Papamarkakis, K. and Laver, N., "Applications of Infrared and Raman Microspectroscopy of Cells and Tissue in Medical Diagnostics: Present Status and Future Promises," Spectroscopy: An International Journal 27(5-6), 463-496 (2012).

[6] Chan, K. L. A. and Kazarian, S. G., "Correcting the effect of refraction and dispersion of light in FT-IR spectroscopic imaging in transmission through thick infrared windows," Analytical chemistry 85(2), 1029-1036 (2013).

[7] Bird, B., Miljković, M., Remiszewski, S., Akalin, A., Kon, M. and Diem, M., "Infrared spectral histopathology (SHP): a novel diagnostic tool for the accurate classification of lung cancer," Laboratory investigation; a journal of technical methods and pathology 92(9), 1358-1373 (2012).

[8] Sun, X., Xu, Y., Wu, J., Zhang, Y. and Sun, K., "Detection of lung cancer tissue by attenuated total reflection-Fourier transform infrared spectroscopy-a pilot study of 60 samples," The Journal of surgical research 179(1), 33-38 (2013).

[9] Wang, X., Shen, X., Sheng, D., Chen, X. and Liu, X., "FTIR spectroscopic comparison of serum from lung cancer patients and healthy persons," Spectrochimica Acta Part A: Molecular and Biomolecular Spectroscopy 122, 193-197 (2014).

[10] Lewis, P. D., Lewis, K. E., Ghosal, R., Bayliss, S., Lloyd, A. J., Wills, J., Godfrey, R., Kloer, P. and Mur, L. A. J., "Evaluation of FTIR spectroscopy as a diagnostic tool for lung cancer using sputum," BMC Cancer 10(1), 640-640 (2010).

[11] Pijanka, J., Sockalingum, G. D., Kohler, A., Yang, Y., Draux, F., Parkes, G., Lam, K.-P., Collins, D., Dumas, P., Sandt, C., van Pittius, D. G., Douce, G., Manfait, M., Untereiner, V. and Sulé-Suso, J., "Synchrotron-based FTIR spectra of stained single cells. Towards a clinical application in pathology," Laboratory investigation; a journal of technical methods and pathology 90(5), 797-807 (2010).

[12] Yano, K., Ohoshima, S., Shimizu, Y., Moriguchi, T. and Katayama, H., "Evaluation of glycogen level in human lung carcinoma tissues by an infrared spectroscopic method," Cancer letters 110(1-2), 29-34 (1996).

[13] Huang, Z., McWilliams, A., Lui, H., Mclean, D. I., Lam, S. and Zeng, H., "Near-infrared Raman spectroscopy for optical diagnosis of lung cancer," International journal of cancer. Journal international du cancer 107(6), 1047-1052 (2003).

[14] Oshima, Y., Shinzawa, H., Takenaka, T., Furihata, C. and Sato, H., "Discrimination analysis of human lung cancer cells associated with histological type and malignancy using Raman spectroscopy," Journal of Biomedical Optics 15(1), 017009 (2010).

[15] Short, M. A., Lam, S., McWilliams, A., Zhao, J., Lui, H. and Zeng, H., "Development and preliminary results of an endoscopic Raman probe for potential in vivo diagnosis of lung cancers," Optics letters 33(7), 711-713 (2008).

[16] Fischer, A. H., Jacobson, K. A., Rose, J. and Zeller, R., "Paraffin embedding tissue samples for sectioning." CSH protocols 3(5) (2008).

[17] Martin, F. L., Kelly, J. G., Llabjani, V., Martin-Hirsch, P. L., Patel, I. I., Trevisan, J., Fullwood, N. J. and Walsh, M. J., "Distinguishing cell types or populations based on the computational analysis of their infrared spectra," Nature Protocols 5(11), 1748-1760 (2010).

[18] Quaroni, L. and Casson, A. G., "Characterization of Barrett esophagus and esophageal adenocarcinoma by Fouriertransform infrared microscopy," The Analyst 134(6), 1240-1246 (2009).

[19] Rice, T. W. and Bronner, M. P., “The Esophageal Wall,” Thoracic Surgery Clinics 21(2), 299-305 (2011).

[20] Wang, T. D., Triadafilopoulos, G., Crawford, J. M., Dixon, L. R., Bhandari, T., Sahbaie, P., Friedland, S., Soetikno, R. and Contag, C. H., "Detection of endogenous biomolecules in Barrett's esophagus by Fourier transform infrared spectroscopy," PNAS 104(40), 15864-15869 (2007).

[21] Chaudhri, V. K., Salzler, G. G., Dick, S. A., Buckman, M. S., Sordella, R., Karoly, E. D., Mohney, R., Stiles, B. M., Elemento, O., Altorki, N. K. and McGraw, T. E., "Metabolic alterations in lung cancer-associated fibroblasts correlated with increased glycolytic metabolism of the tumor," Molecular Cancer Research 11(6), 579-592 (2013).

[22] Rimessi, A., Patergnani, S., Ioannidi, E. and Pinton, P., "Chemoresistance and Cancer-Related Inflammation: Two Hallmarks of Cancer Connected by an Atypical Link, PKC $\zeta$," Frontiers in oncology 3, 232 (2013).

[23] $\mathrm{Wu}$, Y. and Zhou, B. P., "Inflammation: a driving force speeds cancer metastasis," Cell cycle (Georgetown, Tex.) $8(20), 3267-3273$ (2009). 